\title{
Identification of whirlin domains interacting with espin: A study of the mechanism of Usher syndrome type II
}

\author{
LE WANG $^{1,3^{*}}$, BO WEI ${ }^{2 *}$, XUEQI FU ${ }^{3}$, YUCHEN WANG $^{3}$, YUAN SUI $^{3}$, JUNFENG MA $^{3}$, \\ XIANHUI GONG ${ }^{4}$, JILONG HAO ${ }^{1}$ and SHU XING $^{3}$ \\ ${ }^{1}$ Department of Ophthalmology, First Hospital, Jilin University, Changchun, Jilin 130021; \\ ${ }^{2}$ Department of Neurosurgery, China-Japan Union Hospital, Jilin University, Changchun, Jilin 130033; \\ ${ }^{3}$ Edmond H. Fischer Signal Transduction Laboratory, School of Life Sciences, Jilin University, Changchun, Jilin 130012; \\ ${ }^{4}$ Department of Opthalmology, Eye Hospital of Wenzhou Medical University, Wenzhou, Zhejiang 325027, P.R. China
}

Received April 1, 2019; Accepted August 19, 2019

DOI: $10.3892 / \mathrm{mmr} .2019 .10728$

\begin{abstract}
Usher syndrome is the most common condition of combined blindness and deafness and is classified into three types (USH1-USH3). USH2 is the most commonly diagnosed of all Usher syndrome cases. There are three identified proteins (usherin, GPR98 and whirlin) that form the USH2 complex. Defects in any of these proteins may cause failure in the formation of the USH2 complex, which is the primary cause of USH2. Whirlin is a scaffold protein and is essential for the assembly of the USH2 protein complex. It has been reported that espin is an interacting partner protein for whirlin. However, which fragment of whirlin interacts with espin remains unclear. In the present study, whirlin $\mathrm{N}$ - and C-terminal fragments in the pEGFP-C2 vectors were constructed. The recombinant plasmids were transfected into COS-7 cells to observe the co-localization by confocal laser scanning microscopy. The interactions between whirlin and espin were investigated by co-immunoprecipitation using the 293 cell line. It was demonstated that only the whirlin N-terminal fragment was able to interact with espin and the PR (proline-rich) region in whirlin
\end{abstract}

Correspondence to: Dr Jilong Hao, Department of Ophthalmology, First Hospital, Jilin University, 71 Xinmin Street, Changchun, Jilin 130021, P.R. China

E-mail:52723060@qq.com

Dr Shu Xing, Edmond H. Fischer Signal Transduction Laboratory, School of Life Sciences, Jilin University, 2699 Qianjin Street, Changchun, Jilin 130012, P.R. China

E-mail: xingshu@jlu.edu.cn

"Contributed equally

Abbreviations: USH2, Usher syndrome type II; PDZ, postsynaptic density-95/discs large/zona occludens-1; PR, proline-rich; DMEM, Dulbecco's modified Eagle's medium; DMSO, dimethyl sulfoxide; CoIP, co-immunoprecipitation; EDTA, ethylene diamine triacetic acid; PVDF, polyvinylidene fluoride

Key words: USH2, whirlin, espin, $\mathrm{PR}$ regions, interaction may be important for the interaction. However, the present study did not investigate the interaction between whirlin and espin without the PR domain which warrants future research. Our findings elucidated a primary mechanism of interaction between whirlin and espin, which are crucial for further study on the USH2 complex and USH2 pathogenesis.

\section{Introduction}

Usher syndrome is a disease affecting both vision and hearing, occurring in about 1 in 23,000 individuals worldwide (1-3). Usher syndrome has three clinical types. Type I (USH1) is manifested as congenital profound deafness as well as vestibular dysfunction; USH2 presents as congenital moderate hearing loss and normal vestibular function; and USH3 is characterized by progressive hearing impairment and occasional vestibular dysfunction. USH2 is the most common form accounting for approximately $70 \%$ of all cases. Genetic defects in three genes [USH2A (4), USH2C (5), and $U S H 2 D(6)]$ are known to underlie this type of Usher syndrome. There are three identified proteins (usherin, GPR98 and whirlin) that co-localize and form a complex (USH2 complex) in vivo (7-10). Whirlin is the key protein in the USH2 complex, which recruits other USH2 causative proteins at the periciliary membrane in photoreceptors and the ankle link of the stereocilia in hair cells. It has been reported that defects in any of the three proteins may cause mislocalization of the other two proteins and defects in the USH2 complex, which are the primary cause for USH2 pathogenesis (8-13). However, the biological function of the USH 2 complex is largely unknown.

Studies suggest that whirlin is a scaffold protein and may be essential for the assembly of the USH2 complex $(6,14)$. Therefore, it is critical to identify proteins that interact with whirlin and that are part of the USH2 complex (15-18). Reports indicate that whirlin interacts with several proteins other than usherin and GPR98, such as myosin XVa, Eps8 and SANS (19-23). However, evidence to support that any of these proteins are a component of the USH2 complex is still lacking. There are three PDZ (postsynaptic density-95/discs large/zona occludens-1) domains and a proline-rich (PR) region in whirlin (Fig. 1). PDZ domains are distributed throughout the protein 
from the N-terminal to C-terminal. The USH2 complex proteins are known to bind to each other in vitro through PDZ domain-mediated interactions $(7,8,10)$.

Espin is a component protein of the USH2 complex and is a candidate gene for Usher syndrome (24-28). Mutations in espin have been shown to cause deafness in humans (24-26). Espin is expressed in vivo in four isoforms resulting from alternative transcription start site and gene splicing (29). Wang et al previously demonstrated that espin is a protein that interacts with whirlin and that espin expression in photoreceptors is altered in whirlin-knockout mice (28). However, which domain of whirlin interacts with espin remains unclear.

In the present study, it was determined that the interaction between whirlin and espin locates at the N-terminal of whirlin. It was shown that a whirlin fragment with the first two PDZ domains and the PR region is sufficient for its interaction with espin. Our findings suggest that the PDZ domain alone is not sufficient for USH2 complex proteins to interact with each other and the PR region might be required for protein stability.

\section{Materials and methods}

DNA plasmids. Whirlin N- and C-terminal fragments (3-693 amino acids and 693-907 amino acids, NP_082916) in the pEGFP-C2 vectors were constructed as described previously $(30,31)$. All DNA plasmids constructed in this study were confirmed by DNA sequencing. Whirlin full-length cDNA (3-907 amino acids, NP_082916), which was originally cloned from the mouse retina into pEGFP-C2 vectors, was obtained from Dr Jun Yang (University of Utah, Salt Lake City, UT, USA).

Antibodies. A polyclonal rabbit-espin antibody (K-14; sc-133324) was purchased from Santa Cruz Biotechnology, Inc. A rabbit polyclonal-whirlin antibody (25881-1-AP) was obtained from Invitrogen/Thermo Fisher Scientific, Inc. and was biotin-labeled prepared according to the manufacturer's instructions (Invitrogen ${ }^{\mathrm{TM}}$ FluoReporter ${ }^{\mathrm{TM}}$ Mini-Biotin-XX protein Labeling kit; Thermo Fisher Scientific, Inc.). The rabbit and mouse antibodies against GFP (ab6556 and ab1218) were from Abcam. Hoechst dye 33342 (4082) and secondary antibodies (8889) were obtained from Cell Signaling Technology.

Cell culture and cytochalasin D treatment. Cell culture preparation and treatment were performed as described previously by Wang et al with minor modification as follows (28). COS-7 and 293 cells were cultured in Dulbecco's modified Eagle's medium (DMEM) supplemented with $5 \%$ fetal bovine serum (FBS; Invitrogen/Thermo Fisher Scientific, Inc.). Transient transfection was carried out using TurboFect in vitro Transfection Reagent (Thermo Fisher Scientific, Inc.) according to the manufacturer's instructions. Briefly, cells were cultured to $\sim 70 \%$ confluency. One microgram of plasmids was diluted in $100 \mu \mathrm{l}$ of serum-free DMEM. Then $2 \mu \mathrm{l}$ of transfection reagent was added to the diluted DNA and mixed by vortexing. One hundred milliliters of transfection reagent/DNA mixture was added to each well (24-well plate) drop-wise. Cells were collected after incubating at $37^{\circ} \mathrm{C}$ in a $\mathrm{CO}_{2}$ incubator for $36 \mathrm{~h}$.
Before being fixed for confocal microscopy analysis, the cells were treated with cytochalasin D. Cytochalasin D treatment was performed by incubating cells at room temperature for $2 \mathrm{~h}$ in $4 \mu \mathrm{M}$ cytochalasin D/DMEM/5\% FBS, which was freshly prepared from $2 \mathrm{mM}$ cytochalasin D/DMSO (dimethyl sulfoxide) stock solution. Cells treated with DMSO $(1: 1,000)$ in DMEM/5\% FBS under the same condition were used as a negative control for cytochalasin D treatment.

Immunoprecipitation and western blotting. Transfected 293 cells were grown as described above and collected from the culture medium. Cells were lysed by homogenization in lysis buffer [50 mM Tris- $\mathrm{HCl} \mathrm{pH} 8.0,150 \mathrm{mM} \mathrm{NaCl}, 0.5 \%$ Triton X-100, $5 \mathrm{mM}$ ethylene diamine triacetic acid (EDTA), $0.5 \mathrm{mM}$ phenylmethanesulfonyl fluoride, $1 \mathrm{X}$ protease inhibitor and $1 \mathrm{mM}$ DTT] and sonication on ice briefly. The cells were centrifuged at $18,000 \mathrm{x} \mathrm{g}$ for $10 \mathrm{~min}$, and the supernatant (Input) was precleared by incubation with protein $\mathrm{G}$ sepharose (Amersham Biosciences) for $1 \mathrm{~h}$. The precleared supernatant was then incubated with a primary antibody for $3.5 \mathrm{~h}$ and centrifuged at $18,000 \mathrm{x} \mathrm{g}$ for $10 \mathrm{~min}$. The resulting supernatant was incubated with protein $\mathrm{G}$ sepharose for $1 \mathrm{~h}$. After centrifugation at 2,000 $\mathrm{xg}$ for $10 \mathrm{~min}$, the pellet was washed with lysis buffer for three times and then boiled in Laemmli sample buffer. All the procedures were performed at $4^{\circ} \mathrm{C}$. A non-immune rabbit immunoglobulin served as a negative control. Cells without the immunoprecipitation were included as a second negative control.

The various cell lysates or the above immunoprecipitate was separated on a $10 \%$ SDS polyacrylamide gel and transferred to a polyvinylidene fluoride (PVDF) membrane. The resulting PVDF membrane was sequentially subjected to blocking with Tris-buffered saline and Tween 20 (TBST, pH 7.5, $20 \mathrm{mM}$ Tris, $150 \mathrm{mM} \mathrm{NaCl}, 0.1 \%$ Tween 20) containing 1\% BSA (A7906, Sigma-Aldrich; Merck KGaA) for $1 \mathrm{~h}$ at room temperature, and then incubated with the anti-GFP antibody at a 1:1,000 dilution (ab6556, Abcam) overnight at $4^{\circ} \mathrm{C}$ and the anti-rabbit $\mathrm{IgG}$ at a 1:5,000 dilution (\#8889, Cell Signaling Technology) for $2 \mathrm{~h}$ at $37^{\circ} \mathrm{C}$. The protein bands were detected using the chemiluminescent substrate with Alpha Innotech Alpha View software on a FluorChem Q machine (Cell Biosciences, Inc.). The intensities of the protein bands were determined by Image J Software (National Institutes of Health, Bethesda, MD, USA) and normalized using sample loading control bands.

Immunofluorescence staining and co-localization analysis. Cultured cells were fixed in a mixture of methanol and acetone (1:1) at $-20^{\circ} \mathrm{C}$ for $10 \mathrm{~min}$ and rinsed with PBS for $5 \mathrm{~min}$ three times. The fixed cells were then blocked in $5 \%$ goat serum/PBS for $1 \mathrm{~h}$, incubated with a rabbit polyclonal-whirlin antibody (25881-1-AP, Invitrogen/Thermo Fisher Scientific) in 5\% goat serum/PBS at 1:100 dilution at $4^{\circ} \mathrm{C}$ overnight, washed three times with PBS and then incubated with the anti-rabbit IgG (8889, Cell Signaling Technology) at a 1:5,000 dilution and Hoechst 33342 (4082, Cell Signaling Technology) in 5\% goat serum/PBS for $1 \mathrm{~h}$. For staining of espin in cultured cells, A polyclonal rabbit-espin antibody with a 1:100 dilution (K-14; sc-133324, Santa Cruz Biotechnology) was used. The stained cells were viewed and photographed on a confocal laser scanning microscope (Model FV1000; Olympus, Tokyo, Japan). 


\section{PcDNA-espin}

1

\section{4}

\section{AR}

\section{PR}

$P R=W H 2$

$A B M$

PEGFP-c1-whirlin

1

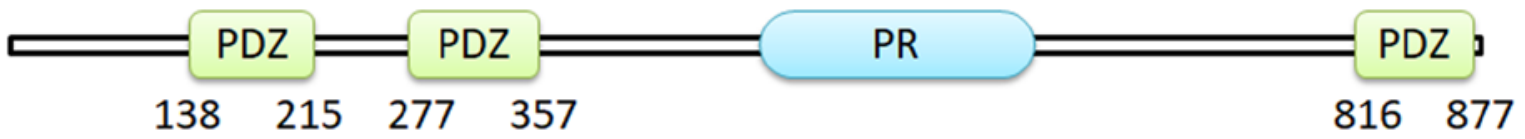

PEGFP-c1-whirlin-n

1

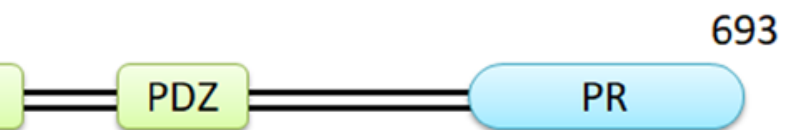

693

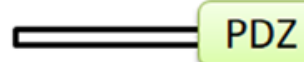

PR

PEGFP-c1-whirlin-c

Figure 1. Schematic diagrams of espin and whirlin domain structure and whirlin fragment constructs. Whirlin has three PDZ domains and a PR region. Whirlin N-terminal fragment (PEGFP-c1-whirlin-n) has PDZ1, PDZ2 and PR, and whirlin C-terminal fragment (PEGFP-c1-whirlin-c) has PDZ3. These fragments were labeled with sequence in the entire gene. Label unit is amino acid. PDZ, postsynaptic density-95/discs large/zona occludens-1; PR, proline-rich.

Ethics statement. This is a basic research study. There were no animals or patients involved in this study. The cell lines used in the in vitro experiments are commercial products, obtained from American Type Culture Collection (ATCC). Moreover, other materials used were normal reagents. There were no ethical issues involved in the study.

\section{Results}

Construction of GFP-tagged whirlin fragment expression plasmids. To identify parts of the whirlin protein required for interaction with espin, two pEGFP-C2 vector derivative plasmids expressing the $\mathrm{N}$-terminal fragment and the $\mathrm{C}$-terminal fragment of the whirlin protein, respectively, were constructed (Fig. 1). The N-terminal fragment contains the first two PDZ domains and the PR region. The C-terminal fragment includes the third PDZ domain. Both plasmids stably expressed whirlin fragments.

Whirlin N-terminal fragment co-localizes with espin. Yang et al and Wang et al reported partial co-localization of whirlin and espin in the retina and the inner ear $(27,28)$. To further elucidate the interaction between whirlin and espin, two whirlin fragments were tested for their abilities to colocalize with full-length espin (Fig. 2). Plasmids expressing GFP-tagged whirlin fragments were cotransfected with mCherry-tagged espin in COS-7 cells. The reason why we used COS-7 cells to study the interaction between whirlin and espin is that the size of COS-7 cells is larger than the majority of other cells, including photoreceptors; thereby, it is more convenient and precise to observe the colocalization of the proteins of interest by confocal laser scanning microscopy. As known to date, whirlin is highly expressed in photoreceptors, while there is little whirlin in the COS-7 cell line. These are the reasons why we chose COS-7 cells as a material to carry out immunofluorescent analysis in vitro and used photoreceptors to perform other functional studies in vivo. In the present study, the whirlin $\mathrm{N}$-terminal fragment was shown to partially co-localize with espin in COS-7 cells. In comparison, the whirlin C-terminal fragment did not appear to colocalize with espin. This suggests that PDZ domains in the whirlin $\mathrm{N}$-terminal fragment may be responsible for whirlin-espin interaction.

Whirlin N-terminal fragment interacts with espin. Co-localization does not necessarily mean interaction between two proteins. In order to confirm that the whirlin N-terminal fragment indeed interacts with espin, we examined the two whirlin fragments for their abilities to interact with full-length espin using a co-immunoprecipitation (CoIP) assay as described in Materials and methods. In our previous study, we tested two different whirlin fragments, where the N-terminal fragment lacked the PR region and the C-terminal fragment contained the PR region, and could not co-immunoprecipitate either whirlin fragment with espin (data not shown). In addition to construct two different fragments, we also modified the CoIP procedure slightly by adding cytochalasin D treatment after co-transfection of whirlin and espin expression plasmids. Cytochalasin D inhibits actin polymerization and induces depolymerization of actin filaments. Although Wang et al (28) found that whirlin exhibited effects on actin bundle network in photoreceptors and hair cells, cytochalasin D treatment here promoted whirlin N-terminal fragment CoIP with espin, but not the whirlin C-terminal fragment (Fig. 3). It appears that with the actin network disrupted, the whirlin N-terminus is able to interact with espin. The results also showed two strong 

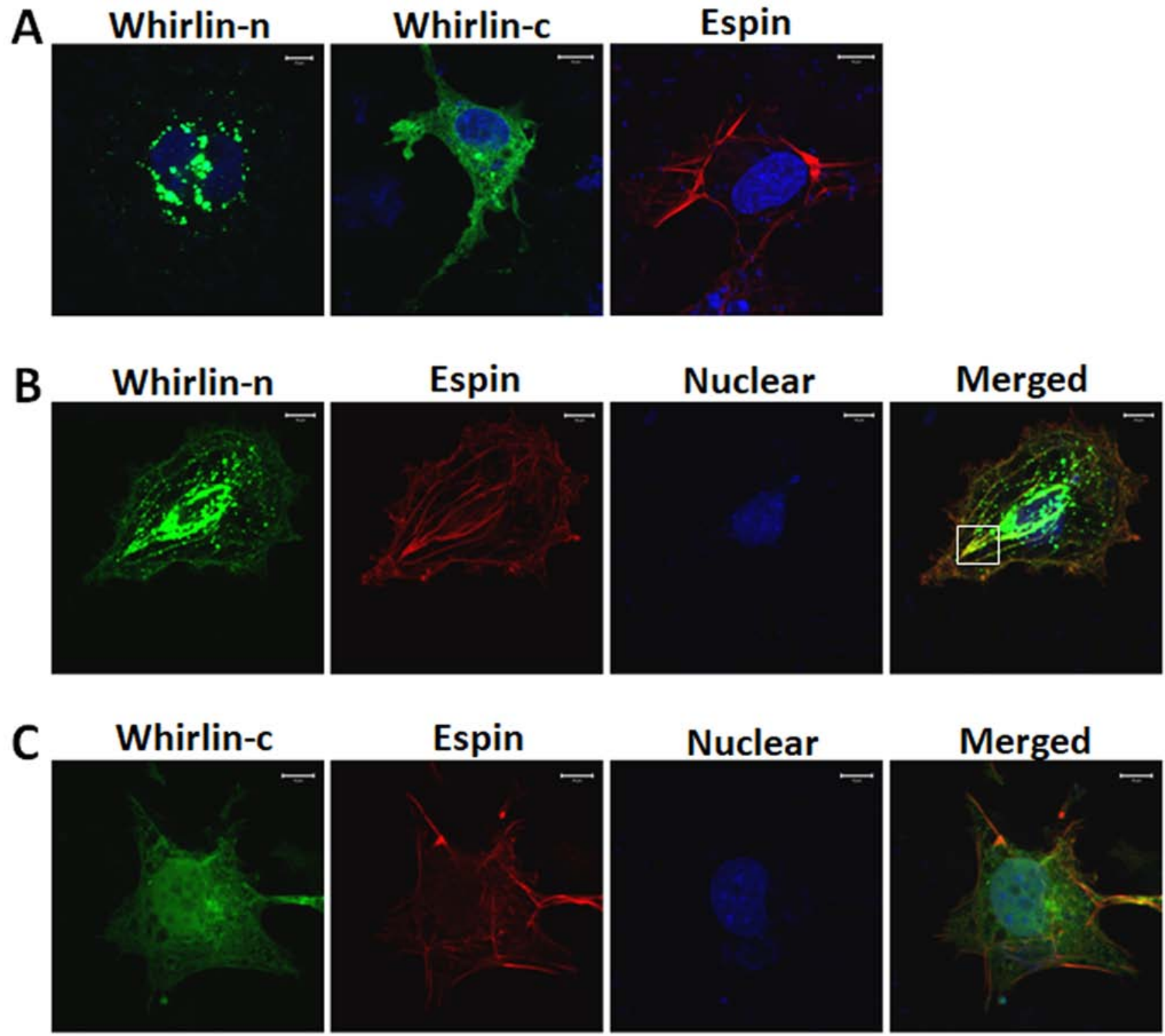

Figure 2. Co-localization of whirlin fragments with espin. Multiple whirlin fragments co-localized with espin in cells as shown by confocal laser scanning microscopy. (A) Distribution of recombinant whirlin N-terminal fragment (whirlin-n) (left), whirlin C-terminal fragment (whirlin-c) (middle) and espin (right) in their respective single-transfected COS-7 cells. (B) GFP-tagged whirlin-n fragment was co-transfected with un-tagged espin in COS-7 cells. The images with un-tagged espin were detected with an anti-rabbit IgG $(\mathrm{H}+\mathrm{L}), \mathrm{F}\left(\mathrm{ab}^{\prime}\right) 2$ fragment (Alexa Fluor ${ }^{\circledR} 594$ conjugate) (Cat. no. \#8889, Cell Signaling Technology) (red). The results showed that the whirlin-n fragment was able to co-localize with espin. Co-localization of GFP-tagged whirlin-n and espin is marked using a white box. (C) No obvious co-localization was observed between GFP-tagged whirlin-c fragment and espin in the double-transfected COS-7 cells, as shown by confocal laser scanning microscopy. The images with un-tagged espin were detected using an anti-rabbit IgG (H+L), F(ab')2 fragment (Alexa Fluor ${ }^{\circledR} 594$ conjugate) (cat. \#8889, Cell Signaling Technology) (red). The data represent the consistent results obtained from at least three independent experiments. Scale bars, $10 \mu \mathrm{m}$.

bands with an approximate molecular weight 50 kilodalton (KDa) in both lanes of IP-GFP whirlin and IP-GFP-whirlin-n. In fact, those strong bands represent the heavy chain of the antibody. The molecular weight of whirlin-c is a little higher than that of the heavy chain. Therefore, whirlin-c only exists in the sample of IP-GFP-whirlin-c.

\section{Discussion}

Whirlin mutations cause retinal degeneration and hearing loss in Usher syndrome type II (USH2) and nonsyndromic deafness $(1-3,6,27)$. It has been shown that whirlin is critical for recruiting other USH2 causative proteins to form a complex at the periciliary membrane complex in photoreceptors and the ankle link of the stereocilia in hair cells $(6,8-12,28)$. However, the biological function and mechanism of the USH2 protein complex are largely unknown. Espin is a component of the USH2 protein complex and is a candidate gene for Usher syndrome. Espin is an actin-binding/bundling protein and may cause human deafness when it is defective (24-26). Wang et al identified that espin interacts with whirlin and crosslinks actin filaments (28). It induces the formation of actin bundles, which are presumed to be stabilized and elongated through prevention from disassembly of these bundles and from depolymerization of actin filaments. The interaction of whirlin with a pool of espin does not bind to actin monomers or filaments, 


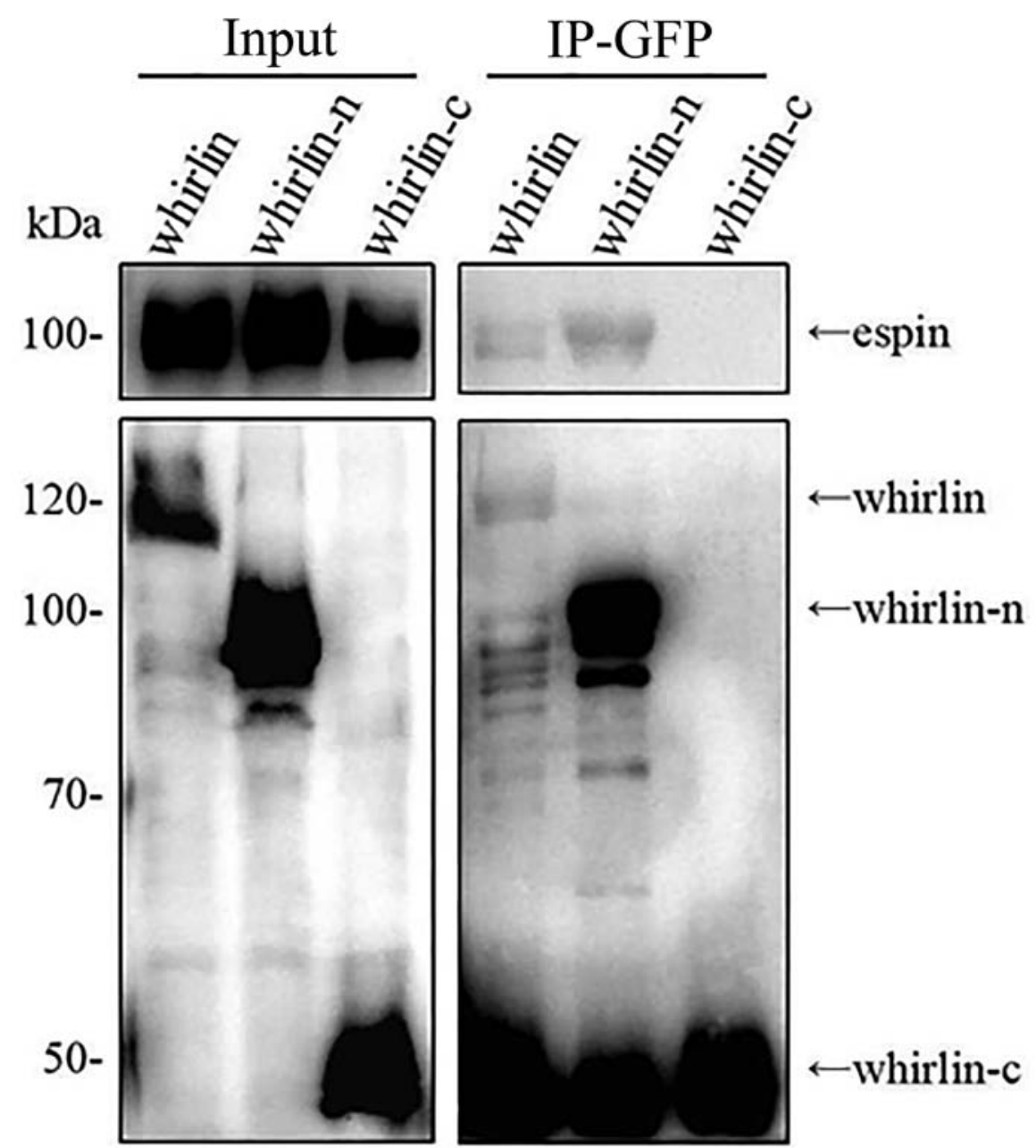

Figure 3. Co-immunoprecipitation of whirlin fragments and espin. Espin was precipitated by whirlin and whirlin N-terminal fragment (whirlin-n) but not the whirlin C-terminal fragment (whirlin-c) after cytochalasin D treatment (upper panels). GFP-tagged whirlin domains (full length, $123.47 \mathrm{kDa}$; whirlin-n, $100.73 \mathrm{kDa}$; and whirlin-c, $49.75 \mathrm{kDa}$ ) are present in the GFP immunoprecipitation from 293T cells double-transfected with GFP-whirlin/whirlin-n/whirlin-c and espin (bottom panels). The western blot of GFP shows the success of the immunoprecipitation procedure, suggesting that whirlin can interact with espin through whirlin-n. The data represent consistent results obtained from at least three independent experiments.

but activates the exchange rate of espin between the pools of actin-free and actin-bound, with the destabilization and shortening of the espin cross-linked actin bundles. In the present study, it was demonstrated that only the whirlin N-terminal fragment was able to interact with espin and that the PR regions in both whirlin and espin proteins are important for their interaction. This finding lays a foundation for further research on the dynamic equilibrium between espin and actin.

The PR regions of whirlin are involved in its interaction with espin. To learn more about the interaction between whirlin and espin, two whirlin fragments were tested for their abilities to interact with full-length espin (Figs. 2 and 3). Previously, we tested two whirlin fragments and did not observe any interaction with espin. We constructed a new set of whirlin fragments to identify their interaction with espin. One distinct difference in the construction is that we moved the PR region into the $\mathrm{N}$-terminal fragment and left the C-terminal fragment with only the third PDZ domain. The interaction between the whirlin N-terminal and espin suggests that the PR region is required for the PDZ domains to interact with espin. PR regions of proteins occur widely in both prokaryotes and eukaryotes. Their functions have been found to be important for protein conformation and sometimes involved in direct binding (32). It is not clear which function the PR regions in whirlin and espin play in whirlin-espin interaction. However, studies have shown that the USH2 complex proteins can interact with each other through PDZ domains, at least in vitro $(7,8,10)$. The requirement of the PR region demonstrated here suggests that the $\mathrm{PR}$ region may be important to enable the truncated whirlin protein to form a conformation that is accessible to espin.

In conclusion, whirlin-espin interaction is important for the architecture of the USH2 complex and actin bundles cross-linked by espin. Our demonstration of whirlin $\mathrm{N}$-terminal fragment interaction with espin, is significantly novel, providing insight into how these two proteins interact to form the USH2 complex. Our findings suggest that although whirlin and espin probably interact through PDZ domains, the PR regions are important for the folding of these proteins into functional conformations. It is crucial to understand the USH2 complex and USH2 pathogenesis. However, the present study did not investigate the interaction between whirlin and espin without the PR domain which warrants further research by our group. 


\section{Acknowledgements}

The authors would like to thank Dr Jun Yang (Utah University, Salt Lake City, UT, USA) for providing the cDNA plasmids.

\section{Funding}

This study was supported by the National Natural Science Foundation of China (81400404 and 31670795); The Project of the Foundation for Changbai Mountain Scholars; Jilin Province Science and Technology Youth Fund (20140520034JH); Young Scholars Program of Norman Bethune Health Science Center of Jilin University (2013205030).

\section{Availability of data and materials}

The datasets used during the present study are available from the corresponding author on reasonable request.

\section{Authors' contributions}

JH, XF and SX designed the study and analyzed data. LW, $\mathrm{BW}, \mathrm{YW}$ and $\mathrm{YS}$ performed the research. JM and XG provided assistance in designing the study and analyzing data. $\mathrm{LW}, \mathrm{JH}$, XF and SX wrote and revised the manuscript. All authors read and approved the final version of the manuscript.

\section{Ethics approval and consent to participate}

This is a basic research study. There were no animals or patients involved in this study. The cell lines used in the in vitro experiments are commercial products, obtained from American Type Culture Collection (ATCC). There were no ethical issues involved in the study.

\section{Patient consent for publication}

Not applicable.

\section{Competing interests}

The authors declare that they have no competing interests.

\section{References}

1. Boughman JA, Vernon M and Shaver KA: Usher syndrome: Definition and estimate of prevalence from two high-risk populations. J Chronic Dis 36: 595-603, 1983.

2. Hartong DT, Berson EL and Dryja TP: Retinitis pigmentosa. Lancet 368: 1795-1809, 2006.

3. Keats BJ and Corey DP: The usher syndromes. Am J Med Genet 89: 158-166, 1999.

4. Eudy JD, Weston MD, Yao S, Hoover DM, Rehm HL, Ma-Edmonds M, Yan D, Ahmad I, Cheng JJ, Ayuso C, et al: Mutation of a gene encoding a protein with extracellular matrix motifs in Usher syndrome type IIa. Science 280: 1753-1757, 1998.

5. Weston MD, Luijendijk MW, Humphrey KD, Möller C and Kimberling WJ: Mutations in the VLGR1 gene implicate G-protein signaling in the pathogenesis of Usher syndrome type II. Am J Hum Genet 74: 357-366, 2004.

6. Ebermann I, Scholl HP, Charbel Issa P, Becirovic E, Lamprecht J, Jurklies B, Millán JM, Aller E, Mitter D and Bolz H: A novel gene for Usher syndrome type 2: Mutations in the long isoform of whirlin are associated with retinitis pigmentosa and sensorineural hearing loss. Hum Genet 121: 203-211, 2007.
7. Adato A, Lefèvre G, Delprat B, Michel V, Michalski N, Chardenoux S, Weil D, El-Amraoui A and Petit C: Usherin, the defective protein in Usher syndrome type IIA, is likely to be a component of interstereocilia ankle links in the inner ear sensory cells. Hum Mol Genet 14: 3921-3932, 2005.

8. Yang J, Liu X, Zhao Y, Adamian M, Pawlyk B, Sun X, McMillan DR, Liberman MC and Li T: Ablation of whirlin long isoform disrupts the USH2 protein complex and causes vision and hearing loss. PLoS Genet 6: e1000955, 2010.

9. Zou J, Chen Q, Almishaal A, Mathur PD, Zheng T, Tian C, Zheng QY and Yang J: The roles of USH1 proteins and PDZ domain-containing USH proteins in USH2 complex integrity in cochlear hair cells. Hum Mol Genet 26: 624-636, 2017.

10. van Wijk E, van der Zwaag B, Peters T, Zimmermann U, Te Brinke H, Kersten FF, Märker T, Aller E, Hoefsloot LH, Cremers CW, et al: The DFNB31 gene product whirlin connects to the Usher protein network in the cochlea and retina by direct association with USH2A and VLGR1. Hum Mol Genet 15: 751-765, 2006.

11. McGee J, Goodyear RJ, McMillan DR, Stauffer EA, Holt JR, Locke KG, Birch DG, Legan PK, White PC, Walsh EJ and Richardson GP: The very large G-protein-coupled receptor VLGR1: A component of the ankle link complex required for the normal development of auditory hair bundles. J Neurosci 26 : 6543-6553, 2006.

12. Michalski N, Michel V, Bahloul A, Lefèvre G, Barral J, Yagi H, Chardenoux S, Weil D, Martin P, Hardelin JP, et al: Molecular characterization of the ankle-link complex in cochlear hair cells and its role in the hair bundle functioning. J Neurosci 27: 6478-6488, 2007.

13. Mathur $P$ and Yang J: Usher syndrome: Hearing loss, retinal degeneration and associated abnormalities. Biochim Biophys Acta 1852: 406-420, 2015.

14. Ciardo MG, Andrés-Bordería A, Cuesta N, Valente P, Camprubí-Robles M, Yang J, Planells-Cases $\mathrm{R}$ and Ferrer-Montiel A: Whirlin increases TRPV1 channel expression and cellular stability. Biochim Biophys Acta 1863: 115-127, 2016.

15. Zou J, Luo L, Shen Z, Chiodo VA, Ambati BK, Hauswirth WW and Yang J: Whirlin replacement restores the formation of the USH2 protein complex in whirlin knockout photoreceptors. Invest Ophthalmol Vis Sci 52: 2343-2351, 2011.

16. Green JA, Yang J, Grati M, Kachar B and Bhat MA: Whirlin, a cytoskeletal scaffolding protein, stabilizes the paranodal region and axonal cytoskeleton in myelinated axons. BMC Neurosci 14: 96, 2013.

17. Chen Q, Zou J, Shen Z, Zhang W and Yang J: Whirlin and PDZ domain-containing 7 (PDZD7) proteins are both required to form the quaternary protein complex associated with Usher syndrome type 2. J Biol Chem 289: 36070-36088, 2014.

18. Mathur PD, Zou J, Zheng T, Almishaal A, Wang Y, Chen Q, Wang L, Vashist D, Brown S, Park A and Yang J: Distinct expression and function of whirlin isoforms in the inner ear and retina: An insight into pathogenesis of USH2D and DFNB31. Hum Mol Genet 24: 6213-6228, 2015.

19. Belyantseva IA, Boger ET, Naz S, Frolenkov GI, Sellers JR, Ahmed ZM, Griffith AJ and Friedman TB: Myosin-XVa is required for tip localization of whirlin and differential elongation of hair-cell stereocilia. Nat Cell Biol 7: 148-156, 2005.

20. Delprat B, Michel V, Goodyear R, Yamasaki Y, Michalski N, El-Amraoui A, Perfettini I, Legrain P, Richardson G, Hardelin JP and Petit C: Myosin XVa and whirlin, two deafness gene products required for hair bundle growth, are located at the stereocilia tips and interact directly. Hum Mol Genet 14: 401-410, 2005.

21. Kikkawa Y, Mburu P, Morse S, Kominami R, Townsend S and Brown SD: Mutant analysis reveals whirlin as a dynamic organizer in the growing hair cell stereocilium. Hum Mol Genet 14: 391-400, 2005.

22. Manor U, Disanza A, Grati M, Andrade L, Lin H, Di Fiore PP, Scita $G$ and Kachar B: Regulation of stereocilia length by myosin $\mathrm{XVa}$ and whirlin depends on the actin-regulatory protein Eps8. Curr Biol 21: 167-172, 2011.

23. Mburu P, Kikkawa Y, Townsend S, Romero R, Yonekawa H and Brown SD: Whirlin complexes with p55 at the stereocilia tip during hair cell development. Proc Natl Acad Sci USA 103: 10973-10978, 2006.

24. Zheng L, Sekerková G, Vranich K, Tilney LG, Mugnaini E and Bartles JR: The deaf jerker mouse has a mutation in the gene encoding the espin actin-bundling proteins of hair cell stereocilia and lacks espins. Cell 102: 377-385, 2000. 
25. Naz S, Griffith AJ, Riazuddin S, Hampton LL, Battey JF Jr, Khan SN, Riazuddin S, Wilcox ER and Friedman TB: Mutations of ESPN cause autosomal recessive deafness and vestibular dysfunction. J Med Genet 41: 591-595, 2004.

26. Donaudy F, Zheng L, Ficarella R, Ballana E, Carella M, Melchionda S, Estivill X, Bartles JR and Gasparini P: Espin gene (ESPN) mutations associated with autosomal dominant hearing loss cause defects in microvillar elongation or organisation. J Med Genet 43: 157-161, 2006.

27. Yang J, Wang L, Song H and Sokolov M: Current understanding of usher syndrome type II. Front Biosci 17: 1165-1183, 2012.

28. Wang L, Zou J, Shen Z, Song E and Yang J: Whirlin interacts with espin and modulates its actin-regulatory function: An insight into the mechanism of Usher syndrome type II. Hum Mol Genet 21: 692-710, 2012.

29. Sekerková G, Zheng L, Loomis PA, Mugnaini E and Bartles JR: Espins and the actin cytoskeleton of hair cell stereocilia and sensory cell microvilli. Cell Mol Life Sci 63: 2329-2341, 2006.
30. Yang J, Liu X, Yue G, Adamian M, Bulgakov O and Li T: Rootletin, a novel coiled-coil protein, is a structural component of the ciliary rootlet. J Cell Biol 159: 431-440, 2002.

31. Chen B, Li A, Wang D, Wang M, Zheng L and Bartles JR: Espin contains an additional actin-binding site in its $\mathrm{N}$ terminus and is a major actin-bundling protein of the Sertoli cell-spermatid ectoplasmic specialization junctional plaque. Mol Biol Cell 10: 4327-4339, 1999

32. Williamson MP: The structure and function of proline-rich regions in proteins. Biochem J 297: 249-260, 1994.

(i) (9) This work is licensed under a Creative Commons Attribution-NonCommercial-NoDerivatives 4.0 International (CC BY-NC-ND 4.0) License. 